REGARDS

SUR L'ECONOMIE ALLEMAND

BULLETIN ECONOMIQUE DU CIRAC
Regards sur l'économie allemande

Bulletin économique du CIRAC

$69 \mid 2004$

Varia

\title{
Priorité à la confiance
}

Isabelle Bourgeois

\section{(2) OpenEdition}

Journals

Édition électronique

URL : http://journals.openedition.org/rea/3263

DOI : 10.4000/rea.3263

ISBN : 978-2-8218-0834-8

ISSN : 1965-0787

Éditeur

CIRAC

Édition imprimée

Date de publication : 1 décembre 2004

Pagination : 3-6

ISSN : 1156-8992

Référence électronique

Isabelle Bourgeois, «Priorité à la confiance », Regards sur l'économie allemande [En ligne], 69| décembre 2004, mis en ligne le 27 janvier 2009, consulté le 15 septembre 2020. URL : http:// journals.openedition.org/rea/3263 


\section{Priorité à la confiance}

La reprise se confirme. Les prévisions formulées par les Instituts de conjoncture dans leur rapport d'automne (publié en octobre) et par le Conseil des Sages dans son rapport annuel (novembre) concordent : en 2004, la croissance devrait s'établir à $1,8 \%$; en 2005 , elle atteindra $1,5 \%$ selon les Instituts et 1,4\% selon le Conseil des Sages. A première vue, la conjoncture semble mollir en 2005 ; or il n'en est rien, expliquent les deux rapports qui insistent sur le fait que la différence a pour seule origine le nombre inférieur de jours ouvrables en 2005 (1,3 en moyenne nationale). Autrement dit : la croissance se maintiendra à l'identique l'an prochain. L'économie allemande est sortie de la phase de stagnation.

Reste une inconnue : l'évolution des prix pétroliers et de la parité $€ / \$$, et son impact sur la croissance mondiale. La publication, en cette mi-novembre, des premières statistiques conjoncturelles du troisième trimestre 2004 a révélé un tassement de la croissance plus net que prévu : par rapport au second, la hausse du PIB n'a été que de $0,3 \%$ dans l'UE 15 comme dans la zone euro, et de $0,1 \%$ en Allemagne. Dès lors, si la flambée des prix pétroliers persiste, que l'euro poursuit son envolée face au dollar et que la conjoncture mondiale perd en vigueur, les prévisions de croissance devront être revues à la baisse - en Allemagne comme dans I'UE.

Même si elle peut perdre en vigueur par rapport aux prévisions, la croissance n'en est pas moins de retour outre-Rhin, la très forte demande mondiale agissant comme un «gigantesque plan de relance », ainsi que l'expliquent les Instituts. Plus que jamais, cette année, l'export aura été l'unique moteur conjoncturel, tournant de surcroît à plein régime : le solde de la balance commerciale devrait augmenter de quelque 30 milliards $€$ en 2004 pour passer à un peu plus de 123 milliards $€$. Les exportations avaient enregistré une hausse de 10,1\% au premier semestre, les prévisions tablent sur le même ordre de grandeur pour l'ensemble de l'année. La spécialisation de l'économie allemande dans le marché mondial a dopé les ventes de biens manufacturés, principalement dans l'automobile et plus encore dans la construction mécanique où l'Allemagne est leader mondial (avant le Japon et les USA) avec une part de marché de 19,3\%.

En valeur, près des deux tiers des ventes s'effectuent toujours à l'intérieur du marché communautaire. Cette 'européanisation' atténue en un sens les effets de la forte appréciation de l'euro sur la balance commerciale. La progression de la part du commerce intra-européen, passée de 19,4\% du PIB en 2002 à 19,9\% en 2003, puis à $21,7 \%$ au cours du premier semestre 2004, est bien moins imputable à la demande émanant des dix nouveaux Etats membres (les exportations vers ces pays ne 'pèsent' qu'entre $2,5 \%$ et 2,9\% du PIB sur la même période) qu'à la solide compétitivité prix des biens et services allemands. Malgré un taux de change $€ / \$$ défavorable sur les marchés tiers, l'Allemagne pourrait ainsi conserver en 2004 le titre de championne du monde de l'export de biens conquis en 2003, estiment les Sept Sages : sa part de marché atteignait alors quelque $10 \%$, presque autant qu'au début des années 1990. Parmi les seuls pays industrialisés, la part de l'Allemagne était même de $16 \%$, le meilleur taux jamais enregistré depuis trente ans.

L'industrie, qui totalisait $90 \%$ des exportations allemandes en 2003, a donc su gagner en compétitivité dans un contexte de concurrence mondiale avivée par l'émergence de nouveaux compétiteurs. Les difficultés récentes de quelques grands groupes-phares (Siemens, VW..., voir REA 68/04) ont quelque peu masqué cet état de fait, entretenant en Allemagne aussi un débat sur les risques de délocalisation, voire de désindustrialisation, avec leur cortège de destruction d'emplois. Le catastrophisme des propos a incité les Sept Sages à analyser la question de plus près. Ils parviennent à la conclusion que le positionnement à l'international de l'industrie allemande révèle que, dans la division mondiale du travail, celle-ci a su tirer profit de sa spécialisation et saisir l'opportunité de l'ouverture de nouveaux marchés. Les entreprises allemandes localisent hors du territoire national la production de biens intermédiaires (les flux d'IDE en témoignent), ce qui leur permet de réduire leurs coûts de production en Allemagne et soutient leur compétitivité prix à l'export.
Fin de la stagnation

Risques : prix pétroliers et parité $€ / \$$

La demande mondiale tire l'économie allemande

Une solide compétitivité prix en Europe et dans le monde

Une industrie dopée par la mondialisation 
L'internationalisation stabilise l'emploi industriel

Le paradoxe allemand : une crise d'adaptation du cadre des activités

Investissements et demande intérieure stagnent encore

Un déficit dans la synchronisation des réformes
Pour schématiser les choix de localisation : les activités intensives en main d'œuvre s'expatrient, celles qui sont intensives en capital demeurent sur le territoire national. Les choix, variables selon les branches, suivent ainsi la diversification des contextes économiques et les mutations globales des activités. La part de l'industrie dans la création de richesse a reculé en 30 ans, mais se stabilise depuis le milieu des années 1990 à un peu plus de $22 \%$. Or si cette tendance au recul de l'industrie par rapport aux services affecte tous les pays industrialisés, l'Allemagne (avec l'Italie) est moins concernée que tous les autres, car elle s'explique aussi par le mouvement d'externalisation de services liés, plus ou moins prononcé selon les pays. L'Allemagne ne souffre pas de désindustrialisation et, contrairement aux apparences, le positionnement international de son industrie a des effets bénéfiques sur l'emploi : indirectement, en diffusant dans l'ensemble du tissu des activités ; directement, en créant de l'emploi. Ainsi, entre 1995 et 2000, les activités industrielles liées à l'export ont généré 300000 emplois, compensant ainsi le recul global de l'emploi industriel (-450 000) lié, lui, à la tertiarisation des activités, ainsi que le rappelle le Conseil des Sages. Et d'expliquer: "Des effets négatifs sur l'emploi, de même qu'une stratégie de compression des coûts et salaires ne sont à attendre que dans la mesure où les entreprises se révèlent incapables de conquérir de nouveaux marchés et de créer des emplois d'un type nouveau ».

La 'mondialisation' n'est donc pas dommageable à l'économie allemande ; loin de là, elle a permis à l'industrie de gagner en compétitivité et de maintenir un certain niveau de croissance économique à l'intérieur du pays. Or si, malgré l'efficience des entreprises, la croissance tendancielle de l'Allemagne ne dépasse guère $1 \%$ depuis de longues années, les raisons en sont à chercher moins dans les variations de la demande mondiale que dans une série de facteurs endogènes qui paralysent l'autre moteur de croissance: la consommation intérieure. Cette analyse est au cœur du rapport des Sept Sages, qu'ils ont de ce fait intitulé «Succès hors des frontières - défis à l'intérieur ». Ils se penchent sur ce qu'il convient d'appeler le 'paradoxe' allemand : alors que l'économie allemande est hautement compétitive si on la considère dans son contexte international, elle l'est nettement moins dès lors qu'on entend par compétitivité " la capacité à hausser le standard de vie domestique ». II s'agit bien des manifestations d'une crise d'adaptation: l'économie a su s'adapter à la globalisation, mais l'adaptation du cadre national régissant les activités et du régime de protection sociale à ces mutations accuse un retard certain.

Instituts et Conseil des Sages font le même constat : si l'export tire la conjoncture et dope les activités industrielles en remplissant les carnets de commande, les investissements bruts en biens d'équipement sont en baisse $(-1,1 \%)$ pour la quatrième année consécutive, malgré un léger mieux au second semestre 2003. Nombre d'entreprises ont été bridées dans leur développement par une politique plus restrictive du crédit bancaire et/ou y ont moins recouru, faute d'une demande suffisante (le secteur bancaire, quant à lui, a surmonté sa crise de restructuration). Elles préfèrent consolider leur bilan ou améliorer le taux d'utilisation de leurs capacités en attendant que la demande intérieure se mette enfin à frémir. La construction mécanique, $3^{\mathrm{e}}$ branche industrielle d'Allemagne après l'automobile et l'électrotechnique, et dont les entreprises (des PME) occupent près de 900000 salariés, fait exception. Elle a connu en septembre un boom des commandes domestiques et vient de rehausser ses prévisions de croissance pour l'année en cours (à $+5 \%$, ce qui sera le meilleur taux enregistré depuis 2000). L'électrotechnique se montre optimiste pour les mêmes raisons, espérant $4 \%$ de croissance en 2004. La consommation privée stagne depuis trois ans, mais donne des signes de frémissement qui pourraient devenir plus nets dès l'an prochain. Les tensions persistantes sur le marché de l'emploi (taux de chômage de 9,3\% selon les critères de I'OIT) comme la faible hausse des salaires bruts (+0,4\% cette année) et des revenus disponibles (+1,8\%) incitent d'autant moins les Allemands à consommer qu'ils n'ont guère confiance en l'avenir : il est vrai que la tendance à la dramatisation des difficultés économiques qu'on peut observer outre-Rhin ne se révèle guère encourageante non plus, et qu'elle est un facteur non négligeable dans la faible croissance intérieure.

Instituts et Conseil des Sages saluent expressément le courage et la persévérance dont a fait preuve le gouvernement fédéral face au mouvement de grogne de l'opinion, ne lâchant pas prise sur la réforme du marché de l'emploi - une réforme struc- 
turelle digne de ce nom. Mais les Instituts incriminent « un grave déficit dans la synchronisation des réformes ». Car le chantier de la protection sociale a pris un retard " qui peut avoir des conséquences dramatiques [si] la substitution des emplois soumis à cotisation sociale par des emplois subventionnés gagne en ampleur ». Car alors, à moins de réduire drastiquement les prestations, le financement des systèmes de protection sociale ne pourra plus être assuré que par la hausse des cotisations ou par un accroissement des transferts du Bund (88 milliards $€$ actuellement, soit $34 \%$ des dépenses fédérales) et donc de la pression fiscale. Cela pèsera forcément sur l'emploi en renchérissant l'emploi salarié, en augmentant la part de l'emploi atypique, et creusera d'autant les déficits des systèmes de protection sociale - un engrenage infernal qu'il convient d'arrêter d'urgence. Instituts et Conseil des Sages formulent des lors des recommandations pour remettre l'économie allemande durablement à flots.

\section{Les réformes Hartz n'auront d'effet sur l'emploi qu'à moyen terme}

Malgré la reprise, la situation sur le marché de l'emploi reste tendue. Si le nombre des actifs occupés a augmenté de 0,2 \% cette année, cela est dû essentiellement à la hausse de l'emploi subventionné (Ich-AG, Mini-Jobs...). Ces mesures n'ont aucune incidence sur l'emploi soumis à cotisation sociale (voir REA 67/04). A l'inverse, la réforme de l'indemnisation du chômage (« Hartz IV », voir REA 68/04) qui entre en vigueur le 01-01-2005 modifiera la structure de la population des chômeurs : quelque 500000 chômeurs touchant actuellement cette Arbeitslosenhilfe qu'abroge la loi Hartz IV ne bénéficieront plus d'aucun soutien dans la mesure où leurs revenus sont considérés comme suffisants. A l'inverse, un nombre équivalent de bénéficiaires de l'aide sociale ancienne manière se verra appliquer le nouveau critère d'employabilité et sera comptabilisé dans les statistiques du chômage. Dans ce contexte, il n'est pas possible actuellement d'établir des prévisions fiables quant à l'évolution du chômage, rappellent tous les experts. II est toutefois probable qu'à partir du mois de février la nouvelle réglementation se traduira par une hausse conséquente du nombre de chômeurs enregistrés ; peut-être même la barre des 5 millions sera-t-elle franchie. Mais une telle hausse ne signifiera en rien une quelconque aggravation de la situation de l'emploi. Car le retour de la croissance, même faible, conjugué aux mesures d'incitation au retour en emploi adoptées dans le cadre des réformes Hartz, la modération salariale des partenaires sociaux et, surtout, la flexibilisation en cours des conditions de travail, devraient apporter à moyen terme une embellie sur le marché du travail allemand.

La première, partagée par les Instituts (sauf le DIW et l'IWH), concerne la consolidation budgétaire, priorité absolue. Cette année encore, l'Allemagne ne respectera pas les critères de Maastricht. Certes, le gouvernement fédéral a entrepris des efforts notables, en réduisant notamment ses dépenses de consommation ; mais ils sont trop timorés. D'un côté, le gouvernement pêche par optimisme, établissant régulièrement son projet de budget sur des prévisions de croissance trop élevées, et donc des attentes irréalistes quant aux recettes fiscales. D'un autre côté, il se trouve face à des Länder qui, eux, ne sont soumis à aucune discipline budgétaire voilà qui plaide une fois de plus pour un Pacte de stabilité national ; les Länder maîtrisent en effet près de la moitié des finances publiques allemandes. Ensuite, les efforts de rigueur du ministre fédéral des Finances sont contrecarrés par les Länder qui font obstruction sur le dossier de la réduction/suppression des subventions ; Instituts et Sages en appellent à leur responsabilité. Dans ce contexte, Bund et Länder doivent aussi corriger les effets pervers d'un soutien trop massif et indifférencié aux économies des Länder est-allemands, dont les gouvernements (sauf celui de la Saxe) ont de surcroît une nette propension à employer des fonds d'investissement à la réduction de leurs déficits budgétaires (10 milliards $€$ selon le Conseil des ministres des Finances allemands).

Globalement, Instituts et Sages préconisent un cap lisible et suivi de consolidation pour ne pas que l'incertitude budgétaire tasse la reprise en pesant sur la confiance, et donc la consommation; et ils prônent (hormis DIW et IWH) le strict respect de l'engagement européen de l'Allemagne sans modification du Pacte de Stabilité. Le Conseil des Sages estime même que l'Allemagne pourrait se rapprocher des $3 \%$, voire les respecter dès 2006, s'il poursuit la consolidation engagée, si les Länder et les communes s'accordent sur une plus stricte discipline budgétaire (ils semblent aujourd'hui prêts à la faire), et si le mode de financement des systèmes de protection sociale est réformé en profondeur. Pour réformer le financement de l'assurance maladie (voir REA 63/03) et de l'assurance dépendance (voir REA 68/04), il conviendrait d'abandonner le principe des cotisations assises sur les salaires au profit d'un financement forfaitaire assis sur tous les revenus et de l'introduction d'éléments de capitalisation. De même, caisses d'assurance privées et publiques devraient être mises en concurrence (une préconisation déjà formulée par le Conseil des Sages en 2002 ; voir REA 59/02).
Consolider d'urgence les finances publiques...

.... en réformant fédéralisme et protection sociale 
Investir dans le capital humain
Il convient aussi d'investir dans l'avenir : en augmentant à $3 \%$ du PIB les dépenses de R\&D (stratégie de Lisbonne) et en accroissant la compétitivité du capital humain grâce à une réforme du système d'éducation et de recherche dont la structure de financement doit être rééquilibrée. Le Conseil des Sages plaide pour une réallocation des ressources en fonction du « bénéfice social » : une plus forte implication du privé (et des études payantes) dans le supérieur, un plus grand engagement public dans le système préscolaire et élémentaire, et le développement d'un segment préscolaire public. Pour préserver le niveau élevé des qualifications sur l'ensemble du territoire, l'Allemagne doit se doter de critères nationaux de qualité et, surtout, l'autonomie des divers types d'établissements doit être renforcée (recrutement, et rémunération des enseignants, contenus des enseignements).

L'OPTINMISME DES INSTITUTS ET DU CONSEIL DES SAgES est délibéré. II se fonde sur un constat objectif : les entreprises sont hautement compétitives ; le gouvernement fédéral a engagé les réformes nécessaires qui porteront bientôt leurs fruits (mais il ne doit pas s'arrêter à mi-chemin). Aux collectivités territoriales et aux partenaires sociaux d'apporter maintenant leur contribution pour asseoir la compétitivité sur une base plus large. Dans cette situation de transition, la priorité absolue est de garder confiance : " quand on voit tout en noir, on finit par ne plus distinguer les voies qu'il est nécessaire et bénéfique de prendre », encourage le Conseil des Sages.

Isabelle Bourgeois (19-11-2004)

Prévisions du rapport d'automne 2004 des instituts de conjoncture

\begin{tabular}{|c|c|c|c|c|c|c|c|}
\hline & \multirow[t]{2}{*}{2003} & \multirow[t]{2}{*}{2004} & \multicolumn{2}{|c|}{2004} & \multirow[t]{2}{*}{2005} & \multicolumn{2}{|c|}{2005} \\
\hline & & & Sem. 1 & Sem. 2 & & Sem. 1 & Sem. 2 \\
\hline \multicolumn{8}{|c|}{ (variation en \% par rapport à la même période de l'année précédente) } \\
\hline $\begin{array}{l}\text { PIB } \\
\text { Est }^{a} \\
\text { Ouest (Berlin inclus) }^{a}\end{array}$ & $\begin{array}{r}-0,1 \\
0,2 \\
-0,1\end{array}$ & $\begin{array}{l}1,8 \\
1,4 \\
1,8\end{array}$ & $\begin{array}{l}1,8 \\
- \\
-\end{array}$ & $\begin{array}{l}1,9 \\
- \\
-\end{array}$ & $\begin{array}{l}1,5 \\
1,5 \\
1,5\end{array}$ & $\begin{array}{r}1,5 \\
- \\
-\end{array}$ & $\begin{array}{r}1,4 \\
- \\
-\end{array}$ \\
\hline $\begin{array}{l}\text { Consommation privée }{ }^{\mathbf{b}} \\
\text { Consommation publique }\end{array}$ & $\begin{array}{l}0,0 \\
0,1\end{array}$ & $\begin{array}{r}0,0 \\
-0,1\end{array}$ & $\begin{array}{r}-0,4 \\
0,1\end{array}$ & $\begin{array}{r}0,3 \\
-0,3\end{array}$ & $\begin{array}{l}0,8 \\
0,0\end{array}$ & $\begin{array}{l}0,7 \\
0,2\end{array}$ & $\begin{array}{r}1,0 \\
-0,3\end{array}$ \\
\hline $\begin{array}{l}\text { Investisssements bruts en biens d'équipt. } \\
\text { Equipement et autres biens } \\
\text { Construction }\end{array}$ & $\begin{array}{r}-2,2 \\
-09 \\
-3,2\end{array}$ & $\begin{array}{l}-1,0 \\
-0,2 \\
-1,7\end{array}$ & $\begin{array}{l}-0,7 \\
-0,6 \\
-0,8\end{array}$ & $\begin{array}{r}-1,3 \\
0,2 \\
-2,5\end{array}$ & $\begin{array}{r}1,5 \\
4,2 \\
-0,8\end{array}$ & $\begin{array}{r}1,6 \\
3,7 \\
-0,3\end{array}$ & $\begin{array}{r}1,5 \\
4,6 \\
-1,2\end{array}$ \\
\hline $\begin{array}{l}\text { Demande intérieure } \\
\text { Exportations } \\
\text { Importations }\end{array}$ & $\begin{array}{r}0,5 \\
1,8 \\
-4,0\end{array}$ & $\begin{array}{r}0,3 \\
10,2 \\
6,7\end{array}$ & $\begin{array}{r}-0,2 \\
10,1 \\
5,6\end{array}$ & $\begin{array}{r}0,7 \\
10,2 \\
7,9\end{array}$ & $\begin{array}{l}1,0 \\
5,9 \\
5,4\end{array}$ & $\begin{array}{l}1,1 \\
6,0 \\
5,9\end{array}$ & $\begin{array}{l}0,8 \\
5,8 \\
4,9\end{array}$ \\
\hline $\begin{array}{l}\text { Revenu national brut } \\
\text { Rev. brut issu du travail salarié } \\
\text { Rev. brut issu des entr. et de la propté. }\end{array}$ & $\begin{array}{l}1,2 \\
0,2 \\
3,5\end{array}$ & $\begin{array}{r}2,9 \\
0,2 \\
11,5\end{array}$ & $\begin{array}{r}3,3 \\
-0,1 \\
14,8\end{array}$ & $\begin{array}{l}2,5 \\
0,4 \\
8,2\end{array}$ & $\begin{array}{l}2,4 \\
1,0 \\
5,9\end{array}$ & $\begin{array}{l}2,2 \\
0,9 \\
3,4\end{array}$ & $\begin{array}{l}2,7 \\
1,2 \\
8,6\end{array}$ \\
\hline Revenu disponible & 1,2 & 1,7 & 1,3 & 2,1 & 2,3 & 2,3 & 2,3 \\
\hline Consommation des ménages & 1,1 & 1,7 & 1,2 & 2,2 & 2,3 & 2,3 & 2,4 \\
\hline Epargne des ménages & 3,3 & 2,6 & 2,7 & 2,5 & 2,4 & 2,9 & 1,8 \\
\hline Prix à la consommation ${ }^{c}$ & 1,1 & 1,6 & - & - & 1,5 & - & - \\
\hline Coûts salariaux unitaires & 0,7 & $-1,3$ & - & - & $-0,3$ & - & - \\
\hline \multicolumn{8}{|c|}{ (état) } \\
\hline $\begin{array}{l}\text { Déficit public en } \% \text { du PIB } \\
\text { Dette publique (en milliards } € \text { ) }\end{array}$ & $\begin{array}{r}-3,8 \\
-81,3\end{array}$ & $\begin{array}{r}-3,8 \\
-82,9\end{array}$ & $\begin{array}{l}- \\
-\end{array}$ & $\begin{array}{l}- \\
-\end{array}$ & $\begin{array}{r}-3,5 \\
-77,6\end{array}$ & - & $\begin{array}{l}- \\
-\end{array}$ \\
\hline Actifs (en millions) & 38,31 & 38,40 & - & - & 38,61 & - & - \\
\hline Est, Berlin inclus (en millions) ${ }^{d}$ & 7,51 & 7,49 & - & - & 7,51 & - & - \\
\hline Ouest (en millions) & 30,75 & 30,85 & - & - & 31,03 & - & - \\
\hline Chômeurs (en millions) ${ }^{d}$ & 4,38 & 4,37 & - & - & 4,33 & - & - \\
\hline Est, Berlin inclus (en millions) & 1,62 & 1,60 & - & - & 1,59 & - & - \\
\hline Ouest (en millions) & 2,75 & 2,77 & - & - & 2,74 & - & - \\
\hline Taux de chômage (en \%) ${ }^{d}$ & 10,3 & 10,2 & - & - & 10,1 & - & - \\
\hline Est, Berlin inclus & 17,8 & 17,6 & - & - & 17,5 & - & - \\
\hline Ouest & 8,2 & 8,2 & - & - & 8,1 & - & - \\
\hline Taux d'épargne & 10,7 & 10,8 & 12,3 & 9,4 & 10,8 & 12,4 & 9,3 \\
\hline
\end{tabular}

Source des données : Rapport d'automne des six instituts de conjoncture DIW (Berlin), HWWA (Hambourg), ifo (Munich), IfW (Kiel), IWH (Halle), RWI (Essen), en date du 15-10-2004. Données établies selon le Système européen des comptes nationaux (SEC 95) ; 2004 et 2005 ; prévisions des Instituts. a) prévisions établies selon les données publiées en avril 2004 ; b) ménages et organismes privés à but non lucratif ; c) $2000=$ indice 100 ; d) données administratives émanant de l'Agence fédérale pour l'emploi. Calculées selon les données OIT, le nombre de chômeurs et le taux de chômage sont inférieurs : ainsi, en 2004, l'Allemagne compte 3,9 millions de demandeurs d'emploi, et le taux de chômage s'établit à 9,3\% (sur la différence entre les deux sources, voir REA 59/02). NB : les données de l'emploi contenues dans ce tableau ne tiennent pas compte des effets induits par l'entrée en vigueur des réformes Hartz. 